\title{
A Layered Architecture for Capacity Planning in Hybrid Networks
}

\author{
U. Datta and L. Lewis \\ Cabletron Systems, Inc. \\ 486 Amherst Street, Nashua, NH 03063 USA \\ Phone 603-337-5737, Fax 603-337-5615, Email udatta@ctron.com
}

\begin{abstract}
An important challenge of network intelligence is capacity evaluation and planning (CEP). CEP is defined typically as follows:

1. monitoring current network characteristics;

2. understanding environmental constraints/considerations;

3. forecasting future needs and technology;

4. evaluating technical opportunity;

5. creating most appropriate, consistent, and coordinated plans on a long, medium, and short term basis;

6. modifying plans based on results of actual implementations in order to provide ongoing cost effective and timely communication services to the users.
\end{abstract}

Here we propose a framework for intelligent CEP. We cast the CEP task into a multilayer architecture, where each layer subsumes the prior layers. In this respect we borrow from the subsumption architecture in the robotics community. Importantly, if a Layer $\mathrm{N}$ task fails there is no interference with the lower-layer tasks. Our layers correspond roughly to (i) current capacity evaluation, (ii) supervised what-if scenarios and capacity evaluation thereof, (iii) unsupervised what-if scenarios, and (iv) automated capacity control.

A goal of our work is to accommodate the evolution from packet-based, to hybrid, to cell-based ATM networks. In addition, our approach is link-centered as opposed to devicecentered, where an evaluation of link capacity is relative to a link's work compared to its contribution to traffic throughput. An evaluation of overall network capacity is a function of evaluations of all link capacities.

\section{Keywords}

Capacity Planning, Performance Management, Change Management 